\title{
Medición del sentido de giro, velocidad y posición angular de un eje mediante encoders
}

Danilo Rairán $^{1} \quad$ José Fonseca ${ }^{2}$

\section{Resumen}

Recibido:

Septiembre 28 de 2009

Aceptado:

Marzo 24 de 2010
En este artículo se presentan algoritmos para la detección del sentido de giro, distintos a los reconocidos en la literatura técnica, y además se estudia la medida de la velocidad y la posición angular por medio de un encoder incremental. El principio básico consiste en utilizar una señal cuasi triangular, en lugar de una onda cuadrada convencional. Se compara la salida del algoritmo de velocidad, el cual es asincrónico, con métodos convencionales, que son sincrónicos; y se concluye que los resultados son satisfactorios. También se verifica el cálculo de la medida de posición, por medio de la comparación con un encoder absoluto.

\section{Abstract}

In this paper we present algorithms for detection of rotation sense, different from current algorithms in technical literature. In addition, measurement of angular position and angular speed is studied. The core of the solution is the use of a quasi-triangular signal, instead of the conventional square wave. The output of the velocity algorithm, which is asynchronic, is compared with conventional methods, which are synchronic. It is concluded that results are satisfactory. It is also verified the calculation of position by means of comparison with an absolute encoder.

Palabras clave: Adquisición de datos, encoder absoluto, encoder incremental, posición angular, velocidad angular.

Key Words: Absolute Encoder, Angular Position, Angular Speed, Data Adquisition, Incremental Encoder.

\footnotetext{
${ }^{1}$ Docente en la Universidad

Distrital Francisco José de Caldas.
} drairan@udistrital.edu.co

${ }^{2}$ Tecnólogo en electricidad. Universidad Distrital Francisco José de Caldas. jmfonseca@correo.udistrital.edu.co 


\section{Introducción}

E n este artículo se mide la velocidad angular y la posición angular, con un encoder absoluto y con otro incremental, los cuales prestan la función de sensores. Sus señales se llevan a Matlab, y allí se implementan algoritmos, los cuales las transforman a valores equivalentes a rpm y grados sexagesimales.

Para sensar la posición y la velocidad angular se tienen muchas clases de sensores, a los cuales se les realizan tratamientos, con el fin de obtener variables continuas o discretas. Uno de ellos es el resolver, en el cual la relación entre señales seno y coseno pasa a ser un valor que indica el ángulo, por ejemplo, a través de un algoritmo en Simulink [1]. También es posible utilizar un sensor capacitivo; en este se traducen las señales a un ángulo, a través de un circuito eléctrico [2].

De otra parte, un encoder es un sensor que convierte la posición angular, que es análoga, a un grupo de bits que indica la posición en forma discreta. Algunos tipos de encoder, por su fabricación, son los ópticos, los capacitivos y los magnéticos. Estos pueden tener formas de onda de salida triangular, cuadrada, senoidal, código Gray o Binario.

De acuerdo con la medición que entregan, un encoder puede ser incremental o absoluto. Los primeros proporcionan una salida relativa a la posición inicial de medición, en su lugar, el absoluto reconoce la posición angular sin importar el momento de activación. Estos últimos pueden ser de 6, 8, 12, 16, 24 ó 360 divisiones por vuelta, en sus presentaciones más corrientes.

En este artículo se estudia una forma nueva de tratar las señales para la medición de la posición angular y velocidad angular, en especial en cuanto a la identificación del sentido de giro, por medio de un encoder incremental o absoluto. Se plantean algoritmos, realizados en Matlab, y basados en las soluciones tradicionales, pero en los cuales se aprovechan las características de una onda cuasi triangular en el enconder incremental, en lugar de la forma cuadrada o la senoidal, que son las convencionales.

Los encodersque se tienen parala realización de este trabajo son dos: uno incremental, de 12 pulsos por vuelta, y el otro, uno absoluto, de referencia ENP101F0082, con 8 divisiones por vuelta. El lector interesado en profundizar sobre la definición y características de estos sensores puede ir a revisar la referencia [3]. Además de los sensores, con el propósito de analizar la influencia de la velocidad en la medición de la posición angular, se seleccionó un motor DC. La referencia del motor es 2342 012CR, con voltaje nominal de $12 \mathrm{~V}$ y potencia de salida de $17 \mathrm{~W}$. Finalmente, como enlace entre el encoder y Matlab, se utiliza la tarjeta de adquisición de datos PCI 6024 E de National Instruments.

\section{Identificación del sentido de giro}

En esta sección se describe el algoritmo con el cual se transforman las señales del encoder incremental, para obtener el sentido de giro de un eje. La esencia del cálculo está en la determinación del ángulo formado por la dupla $(\operatorname{ch} 1(t), \operatorname{ch} 2(t))$ con el origen $(0,0)$, en donde ch1 $(\mathrm{t})$ es el voltaje adquirido de un canal del encoder, y ch2(t) es el voltaje del otro canal, según se explica adelante. Además, se anexan otros bloques, para entregar como resultado un 1 ó -1, según sea el giro del eje horario o contra horario.

La idea, que es original de los autores, es que al graficar las ondas del encoder una versus la otra, se observa un rombo, como se muestra en la figura 1. Si las señales fueran senoidales, el resultado sería un círculo. A medida que la señal cambia en el tiempo, y en función del sentido de giro del eje, que se supone en movimiento, la señal en la figura 1 va apareciendo en sentido horario o contra horario. 
La forma de medir el sentido de giro en la gráfica xy es mediante el cálculo del arco tangente, la cual da como resultado el ángulo $\theta(t)$, y en seguida se calcula la razón de cambio de ese ángulo. Se observó que, en el caso de la prueba que realizaron los autores, cuando el sentido de giro del eje es horario, entonces la razón de cambio es negativa, y en contra horario, es positiva.

En la figura 2, está el diagrama de bloques en el cual se sintetizan las tareas del tratamiento de señales. Al inicio del diagrama, además de lo expuesto en el párrafo anterior, se realiza la adición de una constante, correspondiente al promedio de la onda cuasi triangular.

Figura 1. Medida del sentido de giro del motor. $\operatorname{ch} 1(\mathrm{t})$ es la salida del canal uno del encoder incremental; $\operatorname{ch} 2(t)$, la dos. Si se mide la diferencia de ángulo entre dos instantes en particular, se conocerá el sentido de giro de la señal.

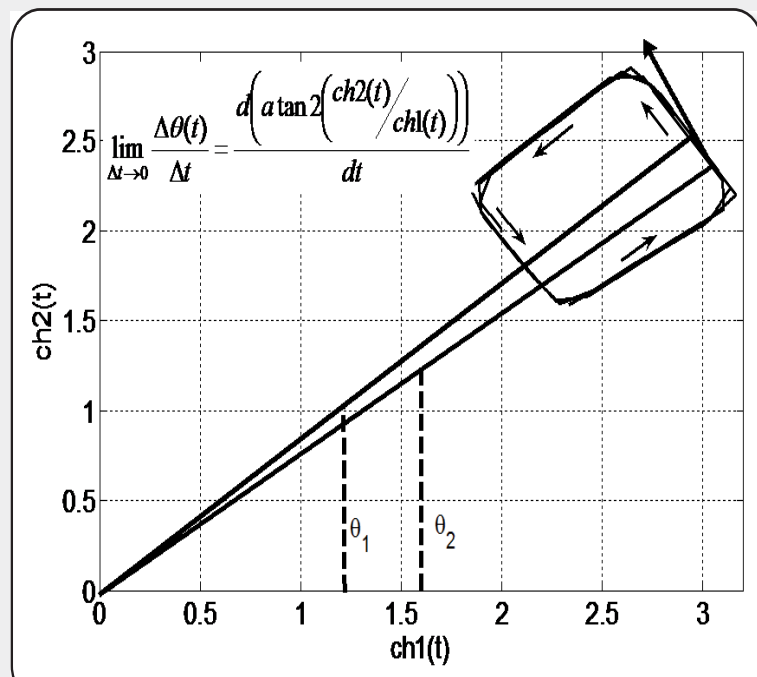

La finalidad de la suma es eliminar la componente continua de la señal, para detectar el cruce por cero. Si bien el cálculo del sentido de giro en la gráfica xy se hace cada $0,1 \mathrm{~ms}$, solo cada cruce por cero se lee la razón de cambio. De esta manera ahora se hace el análisis a espacios iguales y no a tiempos iguales.
Luego de la detección del sentido de giro a espacios iguales se hace una retención de orden cero, esto con el fin de mantener la lectura del signo en un instante hasta el instante siguiente. En seguida se recorta ese valor, y se suma al valor anterior, según se muestra en la figura 2. El último paso es verificar si el resultado es mayor que cero o no. Si es mayor que cero entonces el sentido de giro del eje es positivo, de lo contrario es negativo.

Figura 2. Esquema de la medición del sentido de giro. Inicialmente se calcula la derivada del ángulo. Luego se detecta el mismo punto del ciclo de la señal cuasi triangular. En seguida se aplica una retención de orden cero, y finalmente se detecta el signo.

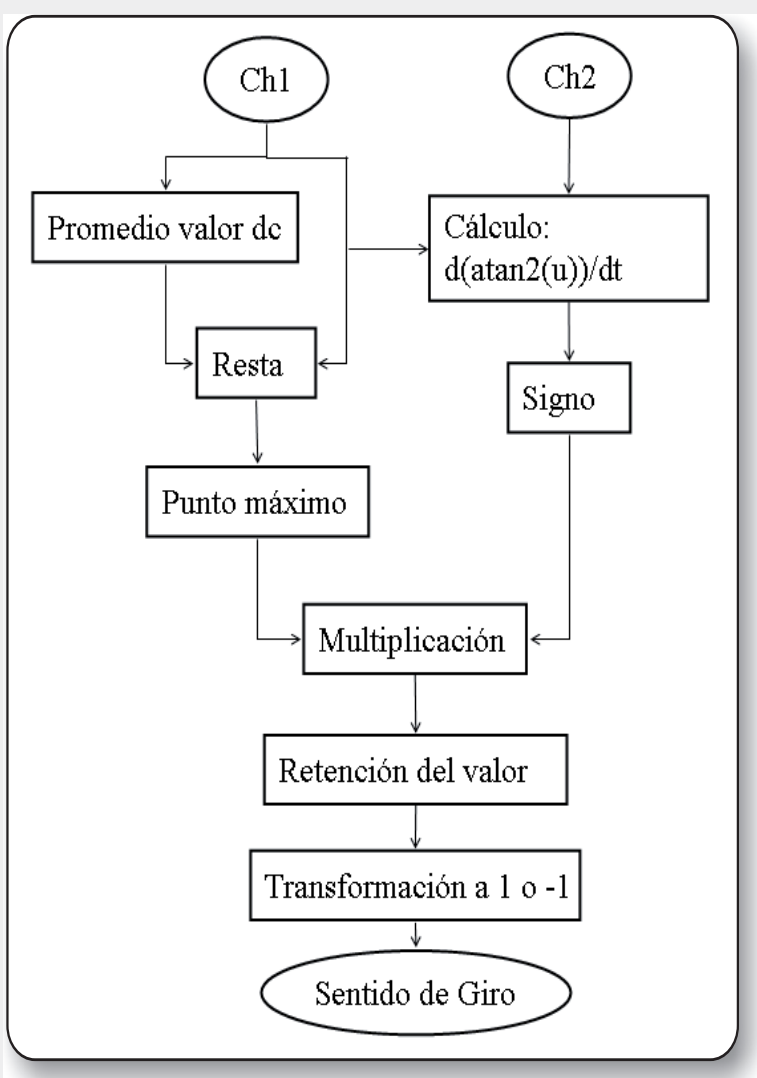

Ahora se explica una estrategia para la identificación del sentido de giro distinta a la de la sección anterior. Aquí la idea principal 
es identificar el ángulo de desfase entre las dos ondas cuasi triangulares, y en función de este desfase definir el sentido de giro. En la figura 3, está el esquema general, en bloques. Se inicia con la transformación de la señal triangular a una señal de pulsos, para lo cual se filtra primero, con el fin de eliminar el ruido de frecuencia alta. Esto se realiza con un filtro tipo Bessel, pasa bajos, de orden 10, con una frecuencia de corte $15 \mathrm{krad} / \mathrm{s}$.

Figura 3. Diagrama de conexiones final para la lectura del signo de la velocidad. La parte uno consiste en la detección de los máximos de las ondas del canal 1 y 2, $(\operatorname{ch} 1(t), \operatorname{ch} 2(t))$. En la parte dos se mide el tiempo entre pulso y pulso. En la final se define si la señal está en adelanto o retraso.

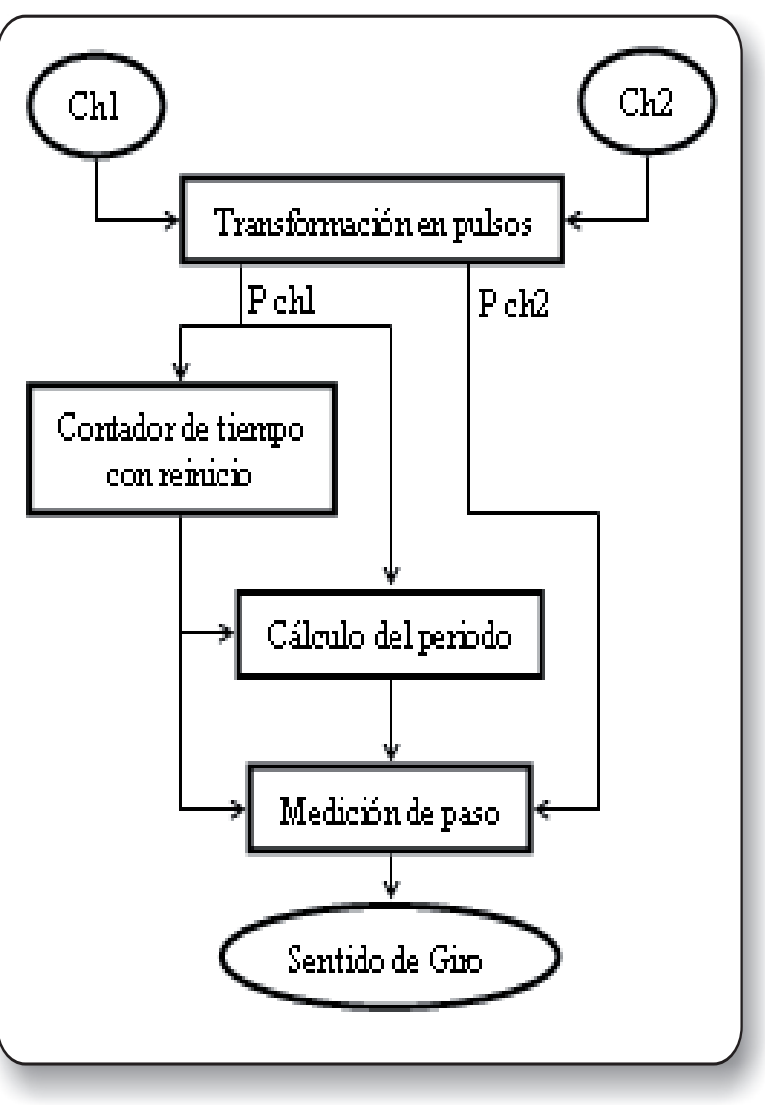

Una vez la señal esta filtrada se deriva, el resultado, dado que es una señal triangular, es un tren de pulsos, correspondientes alos valores donde la pendiente cambia bruscamente. De esos picos solo se toman los que corresponden a los máximos de la señal triangular, como se muestra en la figura 4.
Figura 4. Salida de la parte uno del algoritmo. En la figura se presentan los pulsos detectados correspondientes a los máximos de la señal cuasi triangular. La detección de estos pulsos se dificulta cuando la velocidad es baja y la forma de la onda cambia.

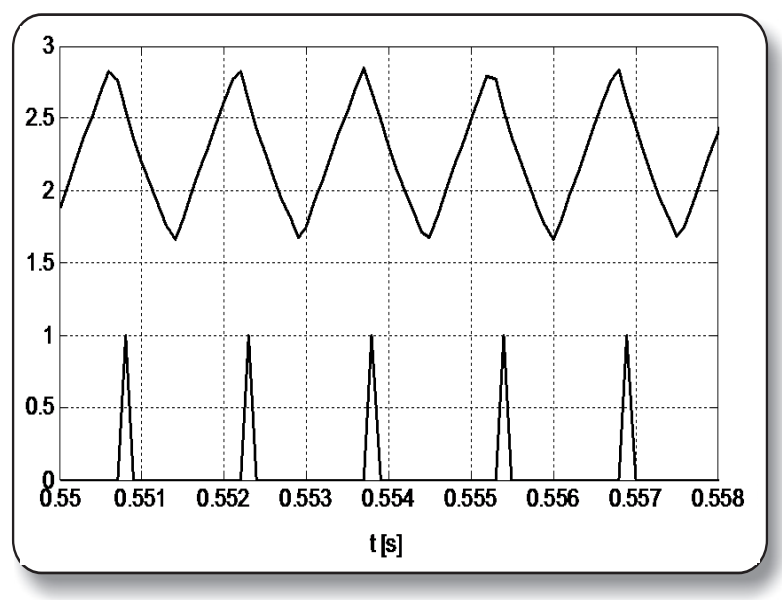

El proceso descrito en el párrafo anterior se repite para el otro canal del encoder, con lo cual se tienen pulsos indicando el instante de tiempo en el cual se presenta la razón de cambio más alta de cada señal triangular. Así, esos pulsos se presentan en proporción con la velocidad del eje. De acuerdo con el sentido de giro del eje uno de los trenes de impulsos está adelante o atrás del otro.

Para saber si el pulso de un canal esta atrás o adelante del pulso del otro canal, se hacen dos lecturas de tiempo. La primera consiste en medir el tiempo entre un pulso y otro de uno de los canales, el cual se considera como referencia. Ese valor corresponde al periodo de la señal cuasi triangular. Se almacena ese valor, y se compara con la segunda lectura de tiempo, la cual corresponda al tiempo entre el pulso que finalizó la medición del periodo del canal de referencia y el inmediatamente siguiente del otro canal.

En las secciones dos y tres se han presentado dos formas de medir el sentido de giro. En la sección que sigue se utiliza este resultado para medir la velocidad angular. 


\section{Medición de la velocidad angular}

El encoder incremental se utiliza para medir la posición o la velocidad angular; sin embargo, es más común en la medida de velocidad [4]. Cuando esta es baja, los algoritmos de tratamiento de señales se hacen indispensables, porque el error de la medida incrementa con la disminución de la velocidad $[5,6]$.

La idea básica del cálculo de la velocidad es medir el periodo de una de las señales cuasi triangulares que se tienen a la salida del encoder, desde Simulink, y buscar la relación que ésta tiene con la velocidad real. El resultado es que tienen una correspondencia inversa, por tal razón es mejor trabajar con el inverso del periodo, es decir, con la frecuencia.

En este trabajo, al graficar la velocidad real del eje, medida con un tacómetro, versus la frecuencia de la señal proveniente de uno de los canales, aparece una línea recta, con el coeficiente de correlación de 0,99, lo cual indica que existe una correlación lineal. La pendiente de esa línea recta es la ganancia del sensor.

Para medir el periodo de la señal triangular se utiliza una fracción del algoritmo con el cual se identifica el sentido de giro. Esto es, la parte que se encarga de encontrar los puntos donde la onda tiene la mayor razón de cambio de la onda, para emitir un pulso en cada caso. El trabajo restante consiste en medir ese tiempo, para lo cual puede utilizarse cualquier tipo de cronómetro.

La contabilización del tiempo puede hacerse, por ejemplo, por la integración de una señal escalón, o por una integración discreta, en un bloque Embedded Matlab Function. La inversión del periodo para lograr la frecuencia, o cualquier operación adicional que se requiera, también puede ser parte del mismo bloque Embedded.
Con el fin de evitar el error que se produce cuando el motor se detiene y no existe un valor siguiente para que el algoritmo calcule un periodo, se agrega una restricción que dice que si el tiempo transcurrido es superior a $0,02 \mathrm{~s}$, entonces la velocidad de salida es cero. Este valor se obtuvo de manera experimental, y depende de cada aplicación en particular.

Los autores pudieron constatar que es importante limitar los resultados con frecuencias superiores a la frecuencia de discretización, que en este caso es de $10 \mathrm{kHz}$, porque son ruido. Por ensayo y error se llegó a que el mejor resultado se consigue con un filtro pasabajos, tipo Bessel, de orden cinco.

Por último se multiplica la lectura de velocidad que se tiene en el momento con el signo. Ese resultado es la velocidad en el eje. En los experimentos realizados la velocidad está entre 0 y 5.000 rpm.

\section{Medición de la posición angular}

La medida de la posición angular por medio del encoder incremental es común en la industria; dado que además de ser un sensor económico, se ha desarrollado gran cantidad de algoritmos, no solo para medir la posición, sino que a partir de esta puede obtenerse la velocidad. Por ejemplo, se utiliza el concepto de derivada pura, o aproximada, a través de cualquier tipo de filtro, como puede verse en los trabajos siguientes [7-13].

La medición de la posición angular consiste en contar los pulsos que entrega el encoder, y relacionar esa cantidad con un número de grados de giro del eje a medir. Una vez más, se utiliza la medición del sentido de giro de la sección tres, con el fin de saber si se suma o se resta cuando llega un pulso. Para el montaje utilizado, cada pulso corresponde a $30^{\circ}$, por tanto se requieren doce pulsos para completar una revolución. 
Tan pronto se encuentra un pulso, este se multiplica por 30 y por el signo en ese momento; luego, ese resultado se le suma al valor de posición anterior. El resultado es la posición en ese instante de tiempo. Como puede entenderse, la medición es relativa al punto en que se comienza a correr el algoritmo. Así, el punto inicial, sin importar la posición angular del eje, es asignada como cero grados.

\section{Implementación con un encoder absoluto}

Si bien el encoder incremental resulta popular para la medida de la posición angular, no se pueden ocultar las desventajas que tiene, como el comportamiento a velocidades bajas, y la imposibilidad para indicar una posición inicial. Aún así, para compensar las deficiencias, algunos investigadores proponen mejoras, por medio de dispositivos mecánicos o por la aplicación de algoritmos, como se presenta en [14].

En este trabajo se utiliza un encoder absoluto, con el fin de comparar y ajustar el comportamiento del incremental, dado que el absoluto entrega lecturas de posición angular de manera precisa. Al respecto la literatura es extensa, y por ejemplo hay trabajos en donde se utiliza el encoder absoluto y se busca incrementar su resolución, como en [15]. Esto, porque aunque entregan una medida con precisión, su resolución es baja, en caso contrario son costosos; por ejemplo, el que se utiliza en este artículo tiene pasos de $45^{\circ}$.

Según se comentó al inicio del artículo, el giro del eje lo realiza un motor DC, el cual tiene acoplado de un lado una caja reductora de velocidad, y de otro el encoder incremental. Así, el incremental mide la velocidad del eje directamente, mientras el absoluto está a la salida de la caja reductora, donde iría la carga del motor. Esto hace que la resolución de $45^{\circ}$, que es baja, sea aún peor, porque la relación en la caja reductora es de 67:1. Por tanto cada paso en el encoder absoluto requiere un poco más de 8,3 revoluciones del eje del motor. Sin embargo es útil con el fin de verificar las mediciones del incremental y para indicar una posición inicial.

En este apartado se transforman las señales digitales que entrega el encoder absoluto, para transformarlas a una lectura de posición angular. Esto implica utilizar al dispositivo mono vuelta como multi vuelta, lo cual se logra con un algoritmo con el esquema en la figura 5. Se inicia negando las salidas digitales del encoder, debido a que el encoder es de lógica negada. En seguida se traduce el código BCD del encoder a grados sexagesimales, en este caso con múltiplos cada $45^{\circ}$, por medio de un bloque Embedded Matlab Function.

Figura 5. Medición de la posición angular con el encoder absoluto. Tp2 es una señal que aparece cada

$45^{\circ}$, EA es el conjunto de señales BCD, las cuales

son 7. En la parte uno, los pulsos se convierten en grados, entre $0^{\circ}$ y $360^{\circ}$. En la parte dos se detecta solo el frente de ascenso de tp2. En la parte tres y cuatro se genera la posición, como un encoder absoluto multivuelta.

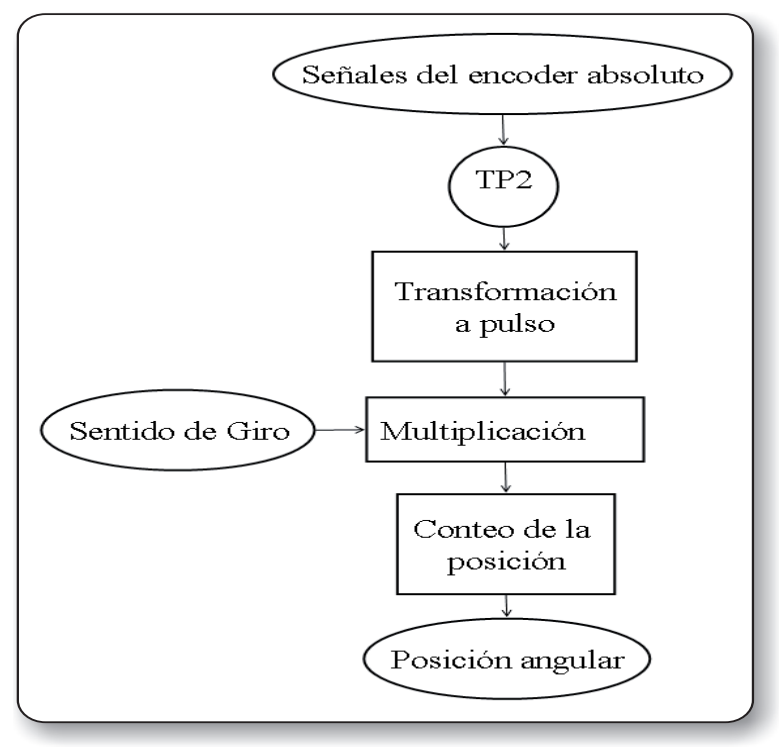

El proceso continúa con la identificación del sentido de giro del eje, en lo cual se utiliza una función en Simulink. Uno de los parámetros de esa función es el valor del voltaje aplicado al motor, con el fin de predecir el signo del primer instante. Se espera que si el voltaje es positivo, el giro sea horario, o contra horario cuando la alimentación sea negativa. 
La lectura del voltaje se hace necesaria porque esperar hasta que existan dos lecturas de posición para identificar el sentido significa esperar un cuarto de giro para el encoder absoluto, lo cual equivale a un poco más de 16 revoluciones del eje del motor.

Las entradas de la función para la identificación del signo ya expuestas son suficientes; sin embargo, se anexan más, con el fin de hacer al algoritmo más robusto. Se incluye un conteo de la cantidad de pulsos medidos, la lectura del ángulo anterior y el sentido de giro en el instante anterior.

La lógica interna en la función de Simulink implica tener en cuenta todas las opciones que pueden presentarse, y no solo el sumar pulsos, o restarlos, de acuerdo con el sentido de giro. Si no que debe tenerse en cuenta, por ejemplo, qué hacer cuando la lectura pasa de $315^{\circ}$ a $0^{\circ}$, esto es cambiar el $0^{\circ}$ por $360^{\circ}$; otro caso es cuando la lectura pasa de $0^{\circ}$ a $315^{\circ}$, en ese caso el resultado es $-45^{\circ}$. Deben tenerse en cuenta todos los casos; uno más es cuando el motor cambia de giro y se repite la lectura de ángulo, etc. El diagrama de flujo general para la detección del sentido de giro se encuentra en la figura 6.

Figura 6. Determinación del sentido de giro para el encoder absoluto. La identificación del sentido no debería hacerse, porque es un encoder absoluto, pero debido a que se pasa de monovuelta a multivuelta, esto se hace necesario.

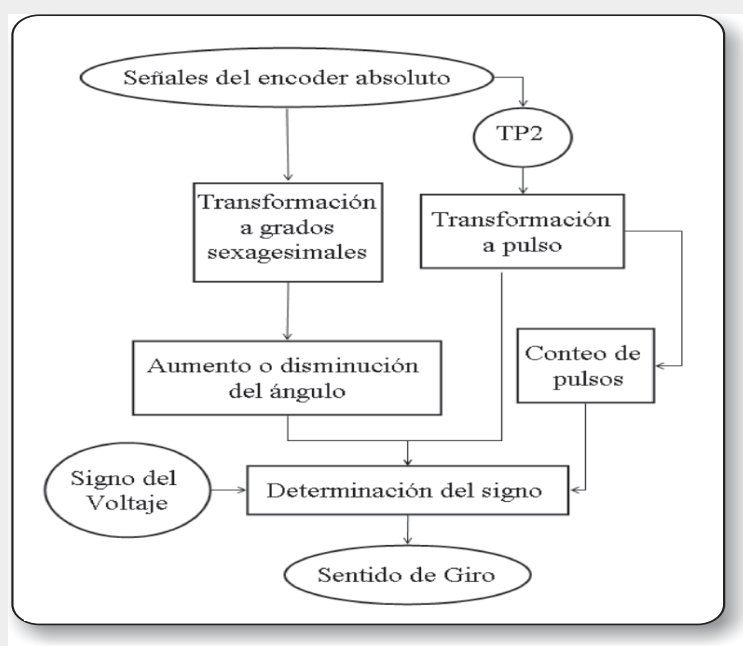

Las indicaciones en los párrafos anteriores convierten al encoder en multi vuelta, entonces, luego de la primera lectura, el signo corresponde a la identificación del signo de la diferencia entre la lectura actual y anterior de posición angular.

\section{Análisis de resultados y discusión}

En aplicaciones como la robótica se utilizan técnicas para el cálculo de la posición angulary velocidad angular, entre las que se encuentran la expansión en series de Taylor, y el método de los mínimos cuadrados, entre otros $[10,11]$. Estos métodos son útiles cuando la lectura de posición se hace a intervalos de tiempo iguales, es decir, para algoritmos sincrónicos, en cuanto al tiempo; mientras que los que aquí se proponen son asincrónicos.

Dada que los métodos de medida sincrónica son muy distintos por su naturaleza a los asincrónicos, se asumen como referencia para calcular el error de medida. Así se verifican los resultados de la aplicación del algoritmo de medida de la sección cuatro, referente a velocidad.

En las ecuaciones 1 a 5 está la medida de velocidad, $\Omega$, a partir de las mediciones de posición, $\theta$. En estas ecuaciones, $\mathrm{k}$ indica el instante actual, k-1 el instante anterior, y Tv el periodo de muestreo. La ecuación 1 es una expansión en series de Taylor de orden uno.

Ladosesdeordendos, segúnlaaproximación de Saíto. De igual manera, la ecuación tres es de orden dos, con la aproximación de Brown. La siguiente, la cuatro, es la aproximación por mínimos cuadrados; mientras que la ecuación 5 presenta el método exacto del cálculo de una derivada [10].

$$
\begin{aligned}
& \Omega_{k}=\frac{2}{T_{v}}\left(\theta_{k}-\theta_{k-1}\right)-\Omega_{k-1} \\
& \Omega_{k}=\frac{11 \theta_{k}-18 \theta_{k-1}+9 \theta_{k-2}-2 \theta_{k-3}}{6 T_{v}}
\end{aligned}
$$


$\Omega_{k}=\frac{13 \theta_{k}-19 \theta_{k-1}+7 \theta_{k-2}-\theta_{k-3}}{8 T_{v}}$

$\Omega_{k}=\frac{3 \theta_{k}-4 \theta_{k-1}+\theta_{k-2}}{2 T_{v}}$

$\Omega_{k}=\frac{\theta_{k}-\theta_{k-1}}{T_{v}}$

La comparación se realiza de la siguiente manera: se asume que el algoritmo en la ecuación 1 es el correcto, es decir, la referencia, y se mide el error, punto a punto, con respecto al resultado con el algoritmo de la sección cuatro. Ese proceso se repite con las ecuaciones 1 a 5 .

El experimento implica el diseño de un perfil de velocidad para el motor, el cual exige que el motor pase de cero a $4.300 \mathrm{rpm}$, en el tiempo inicial, y se mantiene esa referencia por seis segundos, como se observa en la figura 7. Luego se detiene el motor por unos tres segundos; y finalmente, se le da una referencia negativa, en $-4.050 \mathrm{rpm}$, por otros seis segundos.

Con el fin de eliminar los puntos de medida erróneos, por la discretización, se adiciona un filtro pasabajos, tipo Bessel, de orden 5 , con frecuencia de corte $15 \mathrm{rad} / \mathrm{s}$. Este se aplica tanto a la señal que se supone como referencia, como al algoritmo propuesto. El error más grande es de 0,67\%, con el algoritmo de la ecuación 2 como referencia; el menor es 0,65\%, con la ecuación 1 .

Otra verificación del resultado de los algoritmos consiste en comparar el resultado de la medición de la posición angular entre el encoder incremental y el absoluto. Por tanto las medidas son asincrónicas en el tiempo, pero igualmente espaciadas. Para el encoder incremental, cada $30^{\circ}$, y para el absoluto, cada $45^{\circ}$. Sin embargo, téngase en cuanta que entre los dos hay una caja reductora de velocidad, por lo cual es necesario aplicar la relación a una de las dos medidas.

Al igual que el caso de la comparación de la velocidad, se aplica el mismo perfil de velocidad, lo que resulta en una rampa de incremento de posición (giro en sentido horario), a velocidad constante. Se sigue con un tiempo en donde la posición no cambia, porque no se aplica voltaje al motor; para terminar en un giro en sentido contrario al primero, es decir, contra horario. El error absoluto máximo es de $5 \%$, por lo cual puede concluirse que las mediciones pueden ser útiles para algunas aplicaciones industriales.

Figura 7. Verificación de la medida de velocidad angular. La línea blanca delgada corresponde a la salida del algoritmo en la ecuación 1; la línea negra gruesa, bajo la blanca, al cálculo de velocidad mediante el algoritmo asincrónico propuesto en este artículo. A la escala de la imagen puede verse que el resultado es igual.

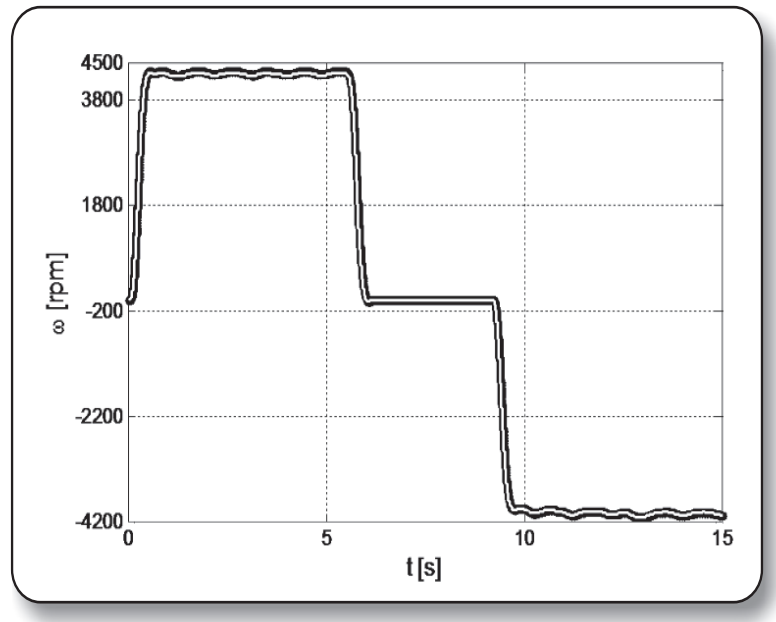

\section{Conclusiones}

En este artículo se presenta el diseño de los algoritmos de medición de velocidad angular, con el encoder incremental, y la medición de posición angular, con los encoders incremental y absoluto. Por el valor del error calculado para los algoritmos se puede afirmar que los resultados son satisfactorios. Por ejemplo, las lecturas entregadas por los algoritmos aquí propuestos se alejan menos de $1 \%$ con respecto a las utilizadas como referencia.

El algoritmo de posición angular con el encoder absoluto tiene la ventaja de ser 
capaz de convertir el encoder monovuelta en multivuelta, con lo cual se aumenta el rango de medida, y se lleva hasta infinito.

La identificación del sentido de giro de un eje es la base de cualquier medida de posición o velocidad angular. En este caso se propone una metodología nueva, y se estudia otra ya propuesta en la literatura técnica. El resultado es el mismo, y pueden ser implementadas según conveniencia.

La idea de alterar la forma de la señal de salida de los canales del encoder incremental fue acertada. Así, se encuentran maneras nuevas de evaluar la frecuencia entre los pulsos del encoder, el desfase, y por tanto la velocidad y posición angular. En este caso, el uso de las señales cuasi triangulares permiten descubrir la utilidad de aspectos que son imposibles de observar en una señal cuadrada o senoidal.

\section{Referencias}

[1] D.W. Brown, D.L. Edwards, G. Georgoulas, B.B. Zhang, G.J. Vachtsevanos, "Real-time fault detection and accommodation for COTS resolver position sensors", Prognostics and Health Management, 2008. PHM 2008. International Conference on, pp $1-6$, Octubre 2008.

[2] P.L. Fulmek, F. Wandling, W. Zdiarsky, G. Brasseur, S.P. Cermak, "Capacitive sensor for relative angle measurement", Instrumentation and Measurement, IEEE Transactions on, pp 1145 - 1149, Diciembre 2002

[3] K. Tobita T. Ohira M. Kajitani C. Kanamori M. Shimojo Aiguo Ming, "A Rotary Encoder Based On MagnetoOptical Storage", Mechatronics, IEEE/ ASME Transactions on, pp 87 - 97, Febrero 2005
N. K. Boggarpu, R. C. Kavanagh, "New Learning Algorithm for High-Quality Velocity Measurement from Low-Cost Optical Encoders", Instrumentation and Measurement Technology Conference Proceedings, 2008. IMTC 2008, IEEE, pp 1908 - 1913.

[5] Akin, B.; Ozturk, S. B.; Niazi, P., Toliyat, H.A., Goodarzi, A., "Low Speed Performance Operation of Induction Motors Drives Using LowResolution Speed Sensor", Industrial Electronics, 2006 IEEE International Symposium on, pp $2110-2115$, Julio 2006.

[6] Se-Han Lee, T.A. Lasky, S.A. Velinsky, "Improved velocity estimation for lowspeed and transient regimes using lowresolution encoders", Mechatronics, IEEE/ASME Transactions on, pp 553 - 560, Septiembre 2004.

[7] Y. Buchnik, R. Rabinovici, "Speed and position estimation of brushless $\mathrm{dc}$ motor in very low speeds", Electrical and Electronics Engineers in Israel, 2004. Proceedings. 2004 23rd IEEE Convention of, pp 317 - 320, Septiembre 2004.

[8] Taeg-Joon Kweon, Dong-Seok Hyun, "High performance speed control of electric machine using Kalman filter and self-tuning regulator", Power Electronics Specialists Conference, 1998. PESC 98 Record. 29th Annual IEEE, vol 1, pp 280 - 286, Mayo 1998.

[9] P. R. Bélanger, "Estimation of angular velocity and acceleration from shaft encoder measurements", Robotics and Automation, 1992. Proceedings., 1992 IEEE International Conference on, vol 1., pp 585 - 592 , Mayo 1992.

[10] M. Benkhoris, M. Ait-Ahmed, "Discrete speed estimation from a position encoder for motor drives", 
Power Electronics and Variable Speed Drives, 1996. Sixth International Conference on (Conf. Publ. No. 429), pp 283 - 287, Septiembre 1996.

[11] R. Brown, S. Schneider, M. Mulligan, "Analysis of algorithms for velocity estimation from discrete position versus time data", Industrial Electronics, IEEE Transactions on, pp $11-19$, Febrero 1992.

[12] G. Liu, "On velocity estimation using position measurements", American Control Conference, 2002. Proceedings of the 2002, vol 2, pp $1115-1120$. Mayo 2002.

[13] K. Hachiya, , T. Ohmae, "Digital speed control system for a motor using two speed detection methods of an incremental encoder", Power Electronics and Applications, 2007 European Conference on, pp $1-10$, Septiembre 2007.

[14] Merry, R. van de Molengraft, R. Steinbuch, M., "Error modeling and improved position estimation for optical incremental encoders by means of time stamping", American Control Conference, 2007. ACC '07, pp 3570 - 3575, Julio 2007.

[15] Wekhande, S. Agarwal, V., "Highresolution absolute position Vernier shaft encoder suitable for highperformance PMSM servo drives", Instrumentation and Measurement, IEEE Transactions on, pp 357 - 364, Febrero 2006. 